University of New Hampshire

University of New Hampshire Scholars' Repository

Space Science Center

Institute for the Study of Earth, Oceans, and

Space (EOS)

2003

\title{
The Development of GRAPE, a Gamma Ray Polarimeter Experiment
}

\author{
Mark L. McConnell \\ University of New Hampshire - Main Campus, mark.mcconnell@unh.edu \\ $\mathrm{J}$ Ledoux \\ University of New Hampshire - Main Campus \\ John R. Macri \\ University of New Hampshire - Main Campus, John.Macri@unh.edu \\ James M. Ryan \\ University of New Hampshire, James.Ryan@unh.edu
}

Follow this and additional works at: https://scholars.unh.edu/ssc

Part of the Astrophysics and Astronomy Commons

\section{Recommended Citation}

The Development of GRAPE, a Gamma Ray Polarimeter Experiment McConnell, M. L. and Ledoux, J. R. and Macri, J. R. and Ryan, J. M., AIP Conference Proceedings, 662, 503-505 (2003), DOI:http://dx.doi.org/ $10.1063 / 1.1579413$

This Conference Proceeding is brought to you for free and open access by the Institute for the Study of Earth, Oceans, and Space (EOS) at University of New Hampshire Scholars' Repository. It has been accepted for inclusion in Space Science Center by an authorized administrator of University of New Hampshire Scholars' Repository. For more information, please contact Scholarly.Communication@unh.edu. 


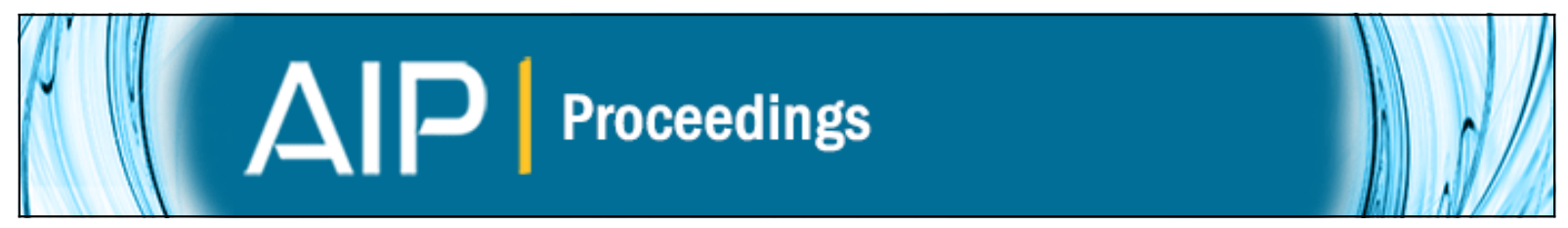

\section{The Development of GRAPE, a Gamma Ray Polarimeter Experiment}

M. L. McConnell, J. R. Ledoux, J. R. Macri, and J. M. Ryan

Citation: AIP Conference Proceedings 662, 503 (2003); doi: 10.1063/1.1579413

View online: http://dx.doi.org/10.1063/1.1579413

View Table of Contents: http://scitation.aip.org/content/aip/proceeding/aipcp/662?ver=pdfcov

Published by the AIP Publishing

\section{Articles you may be interested in}

Development of a graphite polarization analyzer for resonant inelastic x-ray scattering

Rev. Sci. Instrum. 82, 113108 (2011); 10.1063/1.3662472

GammaRay Burst Polarimeter aboard IKAROS

AIP Conf. Proc. 1279, 227 (2010); 10.1063/1.3509271

Prospects for GRB Polarimetry with GRAPE

AIP Conf. Proc. 836, 654 (2006); 10.1063/1.2207970

Development of a hard $\mathrm{x}$-ray polarimeter for gamma-ray bursts

AIP Conf. Proc. 428, 889 (1998); 10.1063/1.55415

Polarimeter for soft $\mathrm{x}$ rays (abstract)

J. Appl. Phys. 81, 5090 (1997); 10.1063/1.364459 


\title{
The Development of GRAPE, a Gamma Ray Polarimeter Experiment
}

\author{
M.L. McConnell*, J.R. Ledoux*, J.R. Macri* and J.M. Ryan* \\ *Space Science Center, University of New Hampshire, Durham, NH 03824
}

\begin{abstract}
.
The measurement of hard X-ray polarization in $\gamma$-ray bursts (GRBs) would add yet another piece of information in our effort to resolve the true nature of these enigmatic objects. Here we report on the development of a dedicated polarimeter design with a relatively large FoV that is capable of studying hard X-ray polarization $(50-300 \mathrm{keV})$ from GRBs. This compact design, based on the use of a large area position-sensitive PMT (PSPMT), is referred to as GRAPE (Gamma-RAy Polarimeter Experiment). The feature of GRAPE that is especially attractive for studies of GRBs is the significant off-axis polarization response (at angles greater than $60^{\circ}$ ). For an array of GRAPE modules, current sensitivity estimates give minimum detectable polarization (MDP) levels of a few percent for the brightest GRBs.
\end{abstract}

\section{INTRODUCTION}

In recent years, largely as a result of the observation of several X-ray, optical and radio afterglows, there has developed a growing consensus that classical $\gamma$-ray bursts (GRBs) are at cosmological distances. Such great distances imply that a typical GRB releases $10^{51}-10^{53} \mathrm{ergs}$ or more within a time span of several seconds.

The general picture that has emerged is one that describes the GRB phenomenom in terms of a relativistic fireball model [e.g., 1]. One common feature of many of these models is that the energy release takes the form of jets that are directed along the rotation axis of the system. Several indirect arguments have been used to argue that such jets are required to explain the observations. For example, the shapes of the observed light curves of some of the observed optical afterglows (e.g., GRB 990510) have been used as supporting evidence for jets related to the external shocks. Since the energy budget of a given GRB depends heavily on assumptions about the extent to which the flow is jet-like, determining the reality and nature of jets in GRBs is becoming an important goal of future observations.

Optical polarization measurements, and the theoretical studies that have been motivated by such measurements, have provided a better insight into the nature of the GRB phenomena. The optical studies, however, probe only the external shock region. In the context of the canonical fireball model, measurements of the hard X-ray polarization during the prompt phase of the GRB promise to provide a similar probe of the internal shock region. Since the out- going flow at the internal shock is expected to be more tightly collimated than the flow at the external shock (resulting from a continuous spreading of the jet as it progresses outward through the fireball), one can perhaps expect a somewhat higher level of hard X-ray polarization (assuming that it arises from synchrotron emission) during the prompt phase of the GRB.

In contrast to the classical GRBs, the prevailing model of soft $\gamma$-ray repeaters (SGRs) involve emission from the vicinity of magnetars, neutron stars with magnetic fields in excess of $10^{14} \mathrm{G}$, with the energy release triggered by massive neutron star crustquakes. Baring [2] suggested that the softness of the events can be attributed to photon splitting in the extremely intense magnetic fields. The photon splitting process degrades the high energy $\gamma$-ray photons to hard X-ray energies [3]. One by-product of photon splitting is that the reprocessed photons would exhibit a polarization level of 25\% [2]. Polarization measurements in the 50-300 keV energy range could therefore provide a test of the importance of photon splitting in SGRs.

\section{A MODULAR POLARIMETER DESIGN}

The basic physical process used to measure linear polarization of hard X-rays (50-300 keV) is Compton scattering. The measurement is based on the fact that the incident photons tend to be scattered at right angles to the incident electric field vector. A Compton scatter po- 


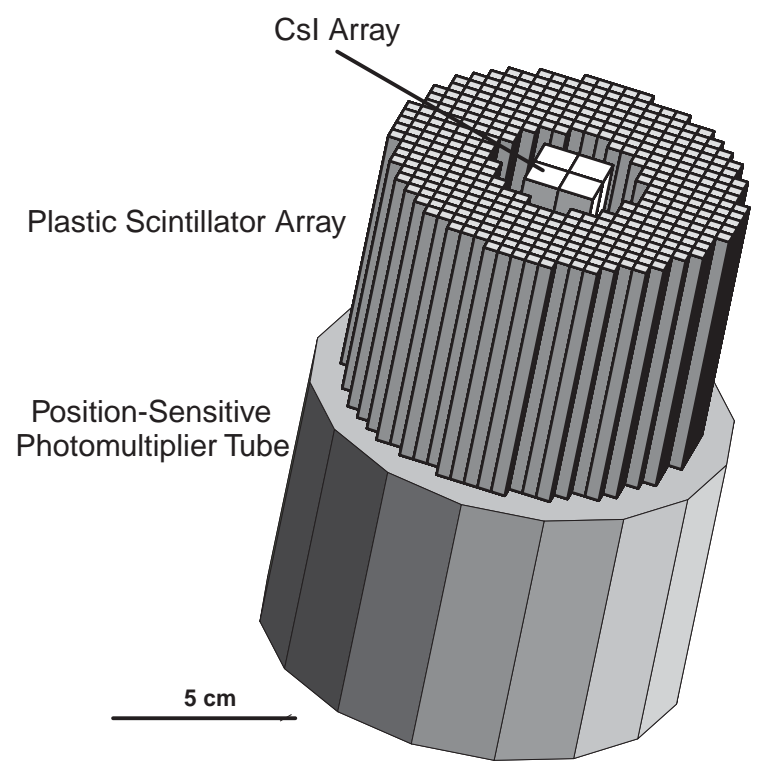

FIGURE 1. Schematic diagram of a polarimeter module.

larimeter consists of two detectors (or detector arrays) that are used to determine the energies of both the scattered photon and the scattered electron. One detector (the scattering detector) provides the medium for the Compton interaction to take place. This detector must be designed to maximize the probability of a single Compton interaction with a subsequent escape of the scattered photon. The primary purpose of the second detector (the calorimeter) is to absorb the full energy of the scattered photon.

We have developed a modular design that places an entire device on the front end of a single 5-inch diameter position-sensitive PMT (PSPMT) [4, 5, 6]. An array of plastic scintillator elements (each $5 \mathrm{~cm}$ long with a cross section of $5 \mathrm{~mm} \times 5 \mathrm{~mm}$ ) provides the spatial resolution in the scattering elements. The spatial resolution is necessary for acurately reconstructing the scattering geometry. The array is arranged in the form of an annulus with an outside diameter of $10 \mathrm{~cm}$. A $2 \times 2$ array of $1 \mathrm{~cm} \mathrm{CsI}$ scintillators is positioned within a well at the center of the array. A multi-anode PMT (MAPMT) provides an independent readout of each CsI element (one element per anode). Independent readouts provide the necessary timing information used to define a Compton scatter event. Figure 1 shows a schematic view of a GRAPE module.

To be recorded as a polarimeter event, an incident photon Compton scatters from one (and only one) of the plastic scattering detectors into the central calorimeter. The incident photon energy can be determined from the sum of the energy losses in both detectors and the azimuthal scattering angle (used to define the polarimetric response) can be determined by the relative locations

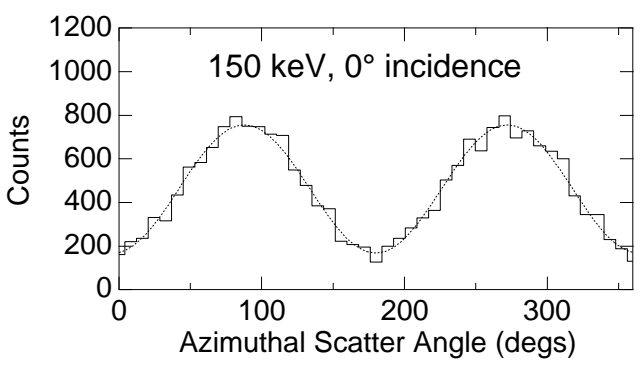

FIGURE 2. The simulated polarimetric response of a GRAPE module. Polarized $150 \mathrm{keV}$ photons vertically incident on the module show an asymmetry in the azimuthal distribution of interactions in the plastic array. The data here have been corrected for intrinsic asymmetries in the module geometry.

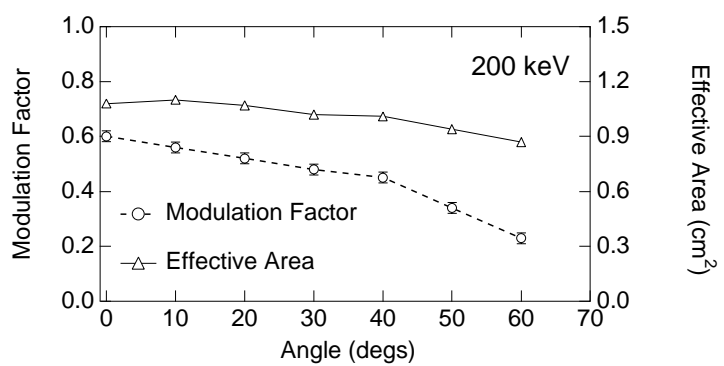

FIGURE 3. The effective area and modulation factor as a function of incidence angle for $200 \mathrm{keV}$ photons (from simulations).

of the plastic and CsI detectors involved in the event. When the polarimeter is arranged so that the incident flux is parallel to the symmetry axis, unpolarized radiation will produce an axially symmetric coincidence rate. If the incident radiation is linearly polarized, then the coincidence rate will show an azimuthal asymmetry whose phase depends on the position angle of the incident radiation's electric vector and whose magnitude depends on the degree of polarization. Figure 2 shows the simulated polarimetric response of a GRAPE module to vertically incident polarized radiation at an energy of 150 $\mathrm{keV}$. The amplitude of the modulation pattern serves to define the level of polarization. The angle at which the modulation pattern is a minimum corresponds to the polarization angle. Figure 3 shows that the module maintains a very good polarimetric response out to very large incidence angles (beyond $60^{\circ}$ ). This makes the GRAPE design especially appealing for GRB studies.

We anticipate that this design would be used in the context of an array of polarimeter modules (a bunch of GRAPEs). Table 1 shows the sensitivity of an array of 36 modules, based on Monte Carlo simulations of this design and reasonable estimates of the background at balloon altitudes. The sensitivity is presented in terms of 
TABLE 1. GRB Polarization Sensitivity (50-300 keV)

\begin{tabular}{cccc}
\hline $\begin{array}{c}\text { Fluence } \\
\left(\mathbf{e r g s ~ c m}^{-2}\right)\end{array}$ & $\begin{array}{c}\text { MDP } \\
\mathbf{T}=\mathbf{1 0} \mathbf{s}\end{array}$ & $\begin{array}{c}\text { MDP } \\
\mathbf{T}=\mathbf{1 0 0} \mathbf{s}\end{array}$ & $\begin{array}{c}\text { Observed Rate } \\
(N>\text { Fluence) }\end{array}$ \\
\hline $1 \times 10^{-4}$ & $2.8 \%$ & $2.9 \%$ & 1 per 320 days \\
$5 \times 10^{-5}$ & $3.9 \%$ & $4.4 \%$ & 1 per 80 days \\
$1 \times 10^{-5}$ & $9.3 \%$ & $13.7 \%$ & 1 per 10 days \\
$5 \times 10^{-6}$ & $14.0 \%$ & $24.4 \%$ & 1 per 6 days \\
$3 \times 10^{-6}$ & $19.4 \%$ & $38.6 \%$ & 1 per 4 days \\
$1 \times 10^{-6}$ & $43.2 \%$ & - & 1 per 2 days \\
\hline
\end{tabular}

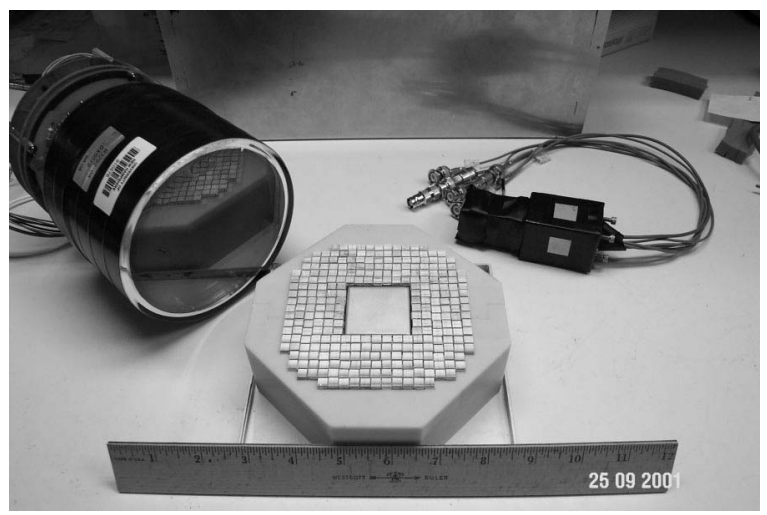

FIGURE 4. The components of the laboratory science model, showing (left to right) the PSPMT, the array of plastic scintillators, and the MAPMT/CsI assembly that fits into the central well of the plastic array.

both the minimum detectable polarization (MDP) and the frequency of observed events above a given fluence level. Note that, for a given fluence level, the MDP is lower for shorter events due to the larger signal-to-background integrated over the event duration. These results suggest that a balloon borne GRAPE would achieve a sensitivity level of a few percent for the strongest GRBs.

\section{LABORATORY SCIENCE MODEL}

We have assembled a laboratory science model (SM) for the purpose of validating the GRAPE design. The components of the SM are shown in Figure 4. The current configuration uses a charge division readout for the PSPMT that provides an event location using only four channels (two readouts for $X$ and two readouts for $Y$ ). During the summer of 2001, we carried out an LED mapping of the PSPMT spatial response using the four channel readout. The LED was stepped through a sequence of locations with a fixed grid spacing of $2.5 \mathrm{~mm}$. The array of measured grid locations is shown in Figure 3. Note the distortions of the grid pattern that are evident near the edge of the PSPMT.

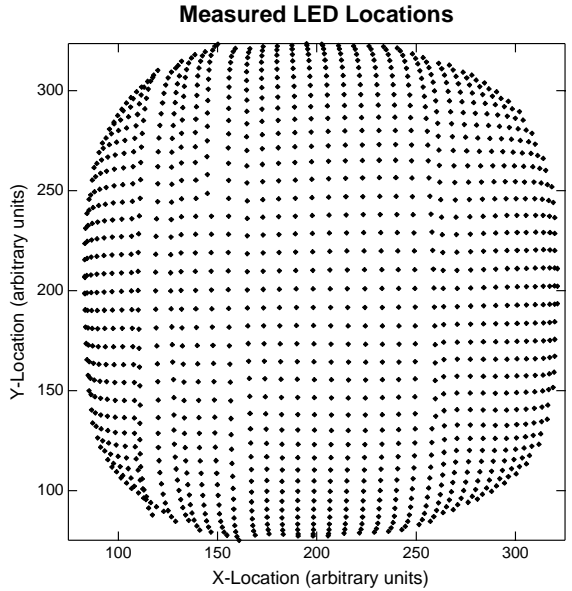

FIGURE 5. Plot of the measured (relative) positions of the LED calibration points.

We have recently started collecting data with the fully assembled science model using a partially polarized beam that is generated by scattering $662 \mathrm{keV}$ photons from a ${ }^{137} \mathrm{Cs}$ source off a block of plastic scintillator. This particular arrangement also provides for the electronic tagging of polarized photons. A preliminary set of uncorrected data shows evidence for a polarization signature. Further processing and analysis of these data are in progress. In the coming months we plan to complete the analysis of laboratory SM data and make detailed comparisons with Monte Carlo simulations. This effort will provide the basis for continued development of a future spacecraft or balloon payload.

\section{ACKNOWLEDGMENTS}

This work is currently supported by NASA grant NAG55324.

\section{REFERENCES}

1. Piran, T., Phys. Rept. 333, 529-533 (2000).

2. Baring, M., ApJ Letters 440, L69-L72 (1995).

3. Baring, M., MNRAS 262, 20-26 (1993).

4. McConnell, M. L., Forrest, D. J., Macri, J., McClish, M., Osgood, M., Ryan, J. M., Vestrand, W. T., and Zanes, C., IEE Trans. Nucl. Sci. 45, 910-914 (1998).

5. McConnell, M. L., Macri, J. R., McClish, M., Ryan, J., Forrest, D. J., and Vestrand, W. T., IEE Trans. Nucl. Sci. 46, 890-896 (1999).

6. McConnell, M. L., Macri, J. R., McClish, M., and Ryan, J., SPIE Conf. Proc. 3764, 70-78 (1999). 